\title{
Glueball-like screening masses in pure SU (3) at finite temperatures
}

\author{
B. Grossmann a, Sourendu Gupta a,b, U.M. Heller ${ }^{c}$, F. Karsch ${ }^{\text {a,d }}$ \\ ${ }^{a}$ HLRZ, c/o KFA Jülich, D-52425 Jülich, Germany \\ ${ }^{b}$ TIFR, Homi Bhabha Road, Bombay 400005, India \\ c SCRI, Florida State University, Tallahassee, FL 32306-4052, USA \\ ${ }^{d}$ Fakultät für Physik, Universität Bielefeld, P.O. Box 100131, D-33501 Bielefeld, Germany \\ and \\ ITP, University of California, Santa Barbara, CA 93106-4030, USA
}

Received 21 September 1993; accepted 14 December 1993

\begin{abstract}
We investigate the finite-temperature excitation spectrum in the gluon sector of SU(3) pure gauge theory through measurements of screening masses in correlations of loop operators. We develop the classification of such operators under the symmetry group of the " $z$-slice". In the confined phase of the theory, we find that the spectrum dynamically realises the zero-temperature symmetries. We observe a large thermal shift of the $0^{++}$ glueball mass. In the deconfined phase, the spectrum distinguishes between operators coupling to electrically and magnetically polarised gluon fields. The former yields a screening mass equal to the Wilson-line screening mass; the latter a method for the measurement of the magnetic mass in the high-temperature limit.
\end{abstract}

\section{Introduction}

The finite temperature deconfinement and chiral symmetry restoration transition in QCD and pure SU (3) gauge theory have been the subject of continuing attention. Many detailed studies of the screening lengths obtained from mesonic and baryonic screening correlation functions have been performed recently [1$3]$. It has become clear that there is a qualitative difference between the hadronic excitation spectra above and below the phase transition temperature $T_{\mathrm{c}}$. For $T<T_{\mathrm{c}}$, the excitation spectrum resembles that at zero temperature--there are (nearly) massless pions, and all the well-known families of hadrons. For $T>T_{\mathrm{c}}$, on the other hand, quarks are liberated. As $T$ increases beyond $T_{c}$, the renormalised quark masses approach those computed in resummed perturbation theory [4], and external currents with mesonic quantum numbers are almost al- 
ways correlated through the exchange of a weakly interacting quark-antiquark pair (three weakly interacting quarks for baryons) [2]. The exception is in the channel with pion quantum numbers. Here the inter-quark interaction remains quite strong even up to $2 T_{\mathrm{c}}$, but various detailed measurements show the absence of a pion pole [3].

Nevertheless, there are strong spatial correlations between quarks [5]. A recent computation [6] relates these correlations to a certain non-perturbative property of the gauge sector of the theory. This is the observation that spatial Wilson loops exhibit area law behaviour at all temperatures [7]. Non-perturbative physics has long been observed for $T \rightarrow T_{c}^{+}$in the pure gauge theory. This suggests that the only non-perturbative behaviour observed in the fermion sector of QCD until now is related to these very same features. In an attempt to further study the gauge sector of the theory, we have performed extensive studies of certain screening correlation functions in the pure gauge theory. In the same way that these are used to study the excitation spectrum in the fermionic sector of QCD, we use them for a detailed study of the excitation spectrum in the pure gauge theory, both above and below $T_{c}$.

Detailed knowledge of one aspect of the pure gauge spectrum is available. In perturbation theory it is possible to compute the gluon propagator. From such a computation of the polarisation tensor, the position of the pole for the electric gluon propagator may be obtained. This is called the electric mass of the gluon, $M_{\mathrm{e}}$. The one-loop result has long been known [8]. For an $\mathrm{SU}\left(N_{\mathrm{c}}\right)$ gauge group with $N_{f}$ (massless) flavours of quarks,

$$
M_{\mathrm{e}}^{2}=c_{\mathrm{e}} g^{2} T^{2}, \quad c_{\mathrm{e}}=\frac{1}{3}\left(N_{\mathrm{c}}+N_{f} / 2\right) .
$$

In this paper we are interested in the case $N_{f}=0$. It should be noted that, at nonzero temperatures, the appearance of an electric or magnetic mass in the gauge field propagator does not violate the usual Ward identities [8]. Furthermore, it has been argued that the pole is a gauge-invariant quantity although defined through a gauge-dependent correlation function [9].

The electric gluon mass gives rise to the phenomenon of Debye screening of colour charges. This is usually seen in the screening of the colour-singlet potential between static quarks and the Debye screening mass can be shown to be close to $M_{\mathrm{e}}$ in perturbation theory [10]. It turns out to be somewhat easier to measure the screening mass in Polyakov line correlations, $M_{\mathcal{P}}$. Again, in perturbation theory, it has been shown that $M_{\mathcal{P}} \approx 2 M_{\mathrm{e}}$ [10]. Close to $T_{\mathrm{c}}, M_{\mathcal{P}}$ has been measured through zero-momentum correlations of Polyakov loops [11]. Since this mass is defined through a gauge-invariant correlation function, it can be given a nonperturbative meaning. It should be emphasised that screening masses of correlations between gauge-invariant objects can only be connected with electric and magnetic gluon masses in perturbation theory. Thus screening masses are more general observables, which in the high-temperature and weak-coupling limit, and only in this limit, may be connected to the gluon electric and magnetic masses. This point has been dealt with more extensively in ref. [10]. 
Although the one-loop computation referred to earlier shows that the magnetic part of the gauge field does not acquire a mass, it is possible to argue that a twoloop evaluation reveals quite the opposite. At finite temperature, therefore, the so-called magnetic mass, $M_{\mathrm{m}}$, must be of the form

$$
M_{\mathrm{m}}=c_{\mathrm{m}} g^{2} T,
$$

where $c_{\mathrm{m}}$ is a dimensionless constant. However, all higher loop order computations contribute to the same order in $M_{\mathrm{m}}$, showing that this quantity cannot be perturbatively evaluated. Thus, the number $c_{\mathrm{m}}$ above is a fully non-perturbative quantity. In this argument we have neglected possible terms in $\ln g$. That these terms can be resummed into a power of $g$ cannot be disproved, and indeed, has been conjectured. There were attempts long ago to measure $M_{\mathrm{m}} / T$ in lattice simulations [12]. We shall demonstrate that one of the screening masses we observe, in the high-temperature limit, goes to $2 M_{\mathrm{m}}$, and hence provides a measurement of $M_{\mathrm{m}}$ in the same way that a measurement of $M_{\mathcal{P}}$ does for $M_{\mathrm{e}}$. In this study we have not been able to analyse the scaling behaviour of $M_{\mathrm{m}}$.

Technically, our measurements are similar to zero-temperature determinations of glueball masses. We construct spatial screening correlators between coloursinglet pure gauge sources which are loops of different shapes. These correlation functions are measured along the $z$-direction at finite temperatures. The transverse slice does not carry representations of the cubic group, as at $T=0$, but of the group $\mathcal{D}_{4}^{\mathrm{h}}$. We describe the representation theory of $\mathcal{D}_{4}^{\mathrm{h}}$, as it applies to our measurements, in section 2 . The corresponding screening masses yield information on the physical excitation spectrum of the high temperature theory in much the same way as do the hadronic screening masses.

The organisation of this paper is the following. In the next section we present the group theory relevant to our measurements. In section 3 we set out details of our simulations and the measurement procedures. The results are collected in the following section and conclusions presented in section 5 .

\section{Symmetries of screening correlators}

In simulations of equilibrium field theories at finite temperatures, the rotational (Lorentz) symmetry of the $T=0$ theory is not realised. This is reflected in practice by the fact that one uses lattices of size $N_{\tau} \times N^{2} \times N_{z}$, where $N_{\tau}<$ $N \leqslant N_{z}$ and $N_{\tau}=1 / a T$ (here $a$ is the lattice spacing). Correlations between operators at different spatial separations measured on such an equilibrium system reveal the spectrum of screening lengths in the theory. Thus, one measures correlation functions of operators separated in the $z$-direction. As a result, it is immaterial whether or not $N=N_{z}$; and, in fact, it may be more convenient to choose $N_{z} \gg N$. In these circumstances, it becomes necessary to identify the symmetry group of the continuum and lattice theories on the " $z$-slices", and perform the decomposition of operators into irreducible representations of this group. This section summarises the results for loop and link operators in a gauge theory. 


\subsection{The symmetry group}

Since the correlations extend in the $z$ direction, the relevant symmetry is of the " $z$-slice". In the infinite volume, continuum limit, the group action is that of the symmetries of a cylinder $\mathcal{C}=O(2) \times\left\{1, \sigma_{z}\right\} . O(2)$ is a non-abelian group with two one-dimensional representations denoted $\mathrm{O}_{1}$ and $\mathrm{O}_{2}$ and two-dimensional representations labeled by the angular momentum $l$ (taking integer values). The $z$ component of the angular momentum can have the two values $\pm l$. The operator $\sigma_{z}$ denotes reflections $t \rightarrow-t$ about the $x-y$ plane, and plays the role of a parity. We denote its eigenvalues, $\{1,-1\}$, by the symbol $P$. The representations of $\mathrm{O}(3)$, describing the zero-temperature glueballs, reduce under $\mathcal{C}$ as

$$
\begin{aligned}
& l_{\mathrm{O}(3)}^{P} \rightarrow 0_{1}^{P}+\sum_{i=1}^{l} i_{C}^{P} \quad \text { for } \quad P=(-1)^{l}, \\
& l_{\mathrm{O}(3)}^{P} \rightarrow 0_{2}^{P}+\sum_{i=1}^{l} i_{C}^{P} \quad \text { for } \quad P=-(-1)^{l} .
\end{aligned}
$$

On a finite lattice the symmetry of the $z$-slice is denoted by $\mathcal{D}_{4}^{\mathrm{h}}=\mathcal{D}_{4} \times\left\{1, \sigma_{z}\right\} . \mathcal{D}_{4}$ is the group of symmetries of a square and has 8 elements in 5 conjugacy classes,

$E$ : identity

$C_{4}: \quad$ two rotations about the $t$-axis by $\pm \pi / 2$

$C_{4}^{2}$ : $\quad$ rotation about the $t$-axis by $\pi$

$\sigma: \quad$ two reflections on the $x-t$ and $y-t$ planes

$\sigma^{*}: \quad$ two reflections on the planes built from the $t$-axis

and the two diagonals in the $x-y$ plane.

$\mathcal{D}_{4}$ has four one-dimensional irreducible representations (irreps $R$ ) $A_{1}, A_{2}, B_{1}$ and $B_{2}$ and one two-dimensional irrep $E$. The labelling of the irreps of the full symmetry group is as $R^{P C}$ where $P$ stands for parity under $\sigma_{z}$ and $C$ for $C$-parity. The lowest few representations of $\mathcal{C}$ reduce under $\mathcal{D}_{4}^{\mathrm{h}}$ as

$$
\begin{array}{lll}
0_{1}^{P} \rightarrow A_{1}^{P}, & 0_{2}^{P} \rightarrow A_{2}^{P}, \\
1^{P} \rightarrow E^{P}, & 2^{P} \rightarrow B_{1}^{P}+B_{2}^{P}, \\
3^{P} \rightarrow E^{P}, & 4^{P} \rightarrow A_{1}^{P}+A_{2}^{P} .
\end{array}
$$

Thus, if the screening masses increase with increasing angular momentum, we find inequivalent $\mathcal{D}_{4}^{\mathrm{h}}$ representations only for $l \leqslant 2$. Higher angular momenta replicate these representations.

In order to compare to lattice data at $T=0$, we also give the operators that transform according to the symmetry group $\mathrm{O}_{\mathrm{h}}$ of a hypercubic lattice in $x, y, t$ space. Rotational symmetry makes this the spatial symmetry group [13]. At any non-zero value of $T$, there is no symmetry argument for the operators and masses to split according to this classification. The reduction $\mathrm{O}_{\mathrm{h}}$ to $\mathcal{D}_{4}^{\mathrm{h}}$ yields 
Table 1

The 4-link operators

\begin{tabular}{|c|c|c|c|c|}
\hline $\mathrm{O}_{\mathrm{h}}$ & $\mathcal{D}_{4}^{\mathrm{h}}$ & $P_{x y}^{4}$ & $P_{x t}^{4}$ & $P_{y t}^{4}$ \\
\hline$A_{1}^{++}$ & $A_{1}^{++}$ & 1 & 1 & 1 \\
\hline \multirow[t]{2}{*}{$E^{++}$} & $A_{1}^{++}$ & -2 & 1 & 1 \\
\hline & $B_{1}^{++}$ & 0 & 1 & -1 \\
\hline \multirow[t]{3}{*}{$T_{1}^{+-}$} & $A_{2}^{+-}$ & 1 & 0 & 0 \\
\hline & $E^{--}$ & 0 & 1 & 0 \\
\hline & & 0 & 0 & 1 \\
\hline
\end{tabular}

Table 2

The twisted 6-link operators

\begin{tabular}{ccrrrr}
\hline $\mathrm{O}_{\mathrm{h}}$ & $\mathcal{D}_{4}^{\mathrm{h}}$ & $T_{x y t}^{6}$ & $T_{x y-t}^{6}$ & $T_{y-x t}^{6}$ & $T_{y-x-t}^{6}$ \\
\hline$A_{1}^{++}$ & $A_{1}^{++}$ & 1 & 1 & 1 & 1 \\
$T_{2}^{++}$ & $B_{2}^{++}$ & 1 & 1 & -1 & -1 \\
& $E^{-+}$ & 1 & -1 & 0 & 0 \\
& $E^{-+}$ & 0 & 0 & 1 & -1 \\
$A_{2}^{+-}$ & $B_{1}^{+-}$ & 1 & 1 & -1 & -1 \\
$T_{1}^{+-}$ & $A_{2}^{+-}$ & 1 & 1 & 1 & 1 \\
& $E^{--}$ & 1 & -1 & 0 & 0 \\
& & 0 & 0 & 1 & -1 \\
\hline
\end{tabular}

$$
\begin{array}{ll}
{\left[A_{1}^{+, C}\right]_{\mathrm{O}_{\mathrm{h}}}=\left[A_{1}^{+, C}\right]_{\mathcal{D}_{4}^{\mathrm{h}}},} & {\left[A_{1}^{-, C}\right]_{\mathrm{O}_{\mathrm{h}}}=\left[A_{2}^{-, C}\right]_{\mathcal{D}_{4}^{\mathrm{h}}}} \\
{\left[A_{2}^{+, C}\right]_{\mathrm{O}_{\mathrm{h}}}=\left[B_{1}^{+, C}\right]_{\mathcal{D}_{4}^{\mathrm{h}}},} & {\left[A_{2}^{-, C}\right]_{\mathrm{O}_{\mathrm{h}}}=\left[B_{2}^{-, C}\right]_{\mathcal{D}_{4}^{\mathrm{h}},},} \\
{\left[E^{+, C}\right]_{\mathrm{O}_{\mathrm{h}}}=\left[A_{1}^{+, C}\right]_{\mathcal{D}_{4}^{\mathrm{h}}}+\left[B_{1}^{+, C}\right]_{\mathcal{D}_{4}^{\mathrm{h}}},} & {\left[E^{-, C}\right]_{\mathrm{O}_{\mathrm{h}}}=\left[A_{2}^{-, C}\right]_{\mathcal{D}_{4}^{\mathrm{h}}}+\left[B_{2}^{-, C}\right]_{\mathcal{D}_{4}^{\mathrm{h}},},} \\
{\left[T_{1}^{+, C}\right]_{\mathrm{O}_{\mathrm{h}}}=\left[A_{2}^{+, C}\right]_{\mathcal{D}_{4}^{\mathrm{h}}}+\left[E^{-, C}\right]_{\mathcal{D}_{4}^{\mathrm{h}}},} & {\left[T_{1}^{-, C}\right]_{\mathrm{O}_{\mathrm{h}}}=\left[A_{1}^{-, C}\right]_{\mathcal{D}_{4}^{\mathrm{h}}}+\left[E^{+, C}\right]_{\mathcal{D}_{4}^{\mathrm{h}},},} \\
{\left[T_{2}^{+, C}\right]_{\mathrm{O}_{\mathrm{h}}}=\left[B_{2}^{+, C}\right]_{\mathcal{D}_{4}^{\mathrm{h}}}+\left[E^{-, C}\right]_{\mathcal{D}_{4}^{\mathrm{h}}}^{\mathrm{h}},} & {\left[T_{2}^{-, C}\right]_{\mathrm{O}_{\mathrm{h}}}=\left[B_{1}^{-, C}\right]_{\mathcal{D}_{4}^{\mathrm{h}}}+\left[E^{+, C}\right]_{\mathcal{D}_{4}^{\mathrm{h}}} .}
\end{array}
$$

\subsection{Representations of loop operators}

In this subsection we list the loop operators required to build the different representations of $\mathcal{D}_{4}^{\mathrm{h}}$. We give all the 4- and 6-link operators, as well as one group of 8-link operators which is required to build the representation $A_{2}^{-+}$. This representation cannot be built out of the shorter loops. The complete list of loops used is 
Table 3

The planar 6-link operators

\begin{tabular}{llrrrrrr}
\hline $\mathrm{O}_{\mathrm{h}}$ & $\mathcal{D}_{4}^{\mathrm{h}}$ & $P_{x x y}^{6}$ & $P_{x x t}^{6}$ & $P_{y y x}^{6}$ & $P_{y y t}^{6}$ & $P_{t t x}^{6}$ & $P_{t t y}^{6}$ \\
\hline$A_{1}^{++}$ & $A_{1}^{++}$ & 1 & 1 & 1 & 1 & 1 & 1 \\
$E^{++}$ & $A_{1}^{++}$ & -2 & 1 & -2 & 1 & 1 & 1 \\
& $B_{1}^{++}$ & 0 & -1 & 0 & -1 & 1 & 1 \\
& & 0 & 1 & 0 & -1 & 1 & -1 \\
$A_{2}^{++}$ & $B_{1}^{++}$ & -1 & 1 & -2 & -1 & -1 & 1 \\
$T_{1}^{+-}$ & $A_{2}^{+-}$ & 1 & 0 & -1 & 0 & 0 & 1 \\
& $E^{--}$ & 0 & -1 & 0 & 0 & 1 & 0 \\
$T_{2}^{+-}$ & & 0 & 0 & 0 & 1 & 0 & -1 \\
& $B_{2}^{+-}$ & 1 & 0 & 1 & 0 & 0 & 0 \\
& $E^{--}$ & 0 & -1 & 0 & 0 & -1 & 0 \\
& & 0 & 0 & 0 & -1 & 0 & -1 \\
\hline
\end{tabular}

Table 4

The bent 6-link operators

\begin{tabular}{|c|c|c|c|c|c|c|c|c|c|c|c|c|c|}
\hline $\mathrm{O}_{\mathrm{h}}$ & $\mathcal{D}_{4}^{\mathrm{h}}$ & $B_{x y t}^{6}$ & $B_{y-x t}^{6}$ & $B_{-x-y t}^{6}$ & $B_{-y x t}^{6}$ & $B_{x t y}^{6+}$ & $B_{x t y}^{6-}$ & $B_{y t-x}^{6+}$ & $B_{y t-x}^{6-}$ & $B_{-x t-y}^{6+}$ & $B_{-x t-y}^{6-}$ & $B_{-y t x}^{6+}$ & $B_{-y t_{\lambda}}^{6-}$ \\
\hline \multirow{3}{*}{$\begin{array}{l}A_{1}^{++} \\
E^{++}\end{array}$} & $A_{1}^{++}$ & 1 & 1 & 1 & 1 & 1 & 0 & 1 & 0 & 1 & 0 & 1 & c \\
\hline & $A_{1}^{++}$ & -4 & -4 & -4 & -4 & 2 & 0 & 2 & 0 & 2 & 0 & 2 & 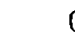 \\
\hline & $B_{1}^{++}$ & 0 & 0 & 0 & 0 & 1 & 0 & -1 & 0 & 1 & 0 & -1 & 0 \\
\hline \multirow[t]{3}{*}{$T_{2}^{++}$} & $B_{2}^{++}$ & 1 & -1 & 1 & -1 & 0 & 0 & 0 & 0 & 0 & 0 & 0 & ( \\
\hline & $E^{-+}$ & 0 & 0 & 0 & 0 & 0 & 1 & 0 & 0 & 0 & -1 & 0 & c \\
\hline & & 0 & 0 & 0 & 0 & 0 & 0 & 0 & 1 & 0 & 0 & 0 & -1 \\
\hline \multirow{3}{*}{$T_{2}^{-+}$} & $B_{1}^{-+}$ & 0 & 0 & 0 & 0 & 0 & 1 & 0 & -1 & 0 & 1 & 0 & -1 \\
\hline & $E^{+-}$ & 1 & -1 & -1 & 1 & 0 & 0 & 1 & 0 & 0 & 0 & -1 & $c$ \\
\hline & & 1 & 1 & -1 & -1 & -1 & 0 & 0 & 0 & 1 & 0 & 0 & 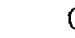 \\
\hline \multirow{3}{*}{$T_{1}^{-+}$} & $A_{1}^{-+}$ & 0 & 0 & 0 & 0 & 0 & 1 & 0 & 1 & 0 & 1 & 0 & 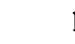 \\
\hline & $E^{++}$ & -1 & 1 & 1 & -1 & 0 & 0 & 1 & 0 & 0 & 0 & -1 & c \\
\hline & & 1 & 1 & -1 & -1 & 1 & 0 & 0 & 0 & -1 & 0 & 0 & c \\
\hline & $A_{2}^{--}$ & 1 & 1 & 1 & 1 & 0 & 1 & 0 & 1 & 0 & 1 & 0 & 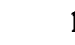 \\
\hline \multirow[t]{2}{*}{$E^{-1}$} & $A_{2}^{-}-$ & 2 & 2 & 2 & 2 & 0 & 1 & 0 & 1 & 0 & 1 & 0 & 1 \\
\hline & $B_{2}^{-}-$ & 0 & 0 & 0 & 0 & 0 & 1 & 0 & -1 & 0 & 1 & 0 & -1 \\
\hline \multirow[t]{3}{*}{$T_{2}^{--}$} & $B_{1}^{--}$ & 1 & -1 & 1 & -1 & 0 & 0 & 0 & 0 & 0 & 0 & 0 & 0 \\
\hline & $E^{+-}$ & 0 & 0 & 0 & 0 & 1 & 0 & 0 & 0 & -1 & 0 & 0 & c \\
\hline & & 0 & 0 & 0 & 0 & 0 & 0 & 1 & 0 & 0 & 0 & -1 & c \\
\hline \multirow[t]{3}{*}{$T_{2}^{+-}$} & $B_{2}^{+-}$ & 0 & 0 & 0 & 0 & 1 & 0 & -1 & 0 & 1 & 0 & -1 & 0 \\
\hline & $E^{-}$ & -1 & 1 & 1 & -1 & 0 & 0 & 0 & 1 & 0 & 0 & 0 & -1 \\
\hline & & -1 & -1 & 1 & 1 & 0 & -1 & 0 & 0 & 0 & 1 & 0 & c \\
\hline \multirow[t]{3}{*}{$T_{1}^{+-}$} & $A_{2}^{+-}$ & 0 & 0 & 0 & 0 & 1 & 0 & 1 & 0 & 1 & 0 & 1 & c \\
\hline & $E^{--}$ & 1 & -1 & -1 & 1 & 0 & 0 & 0 & 1 & 0 & 0 & 0 & -1 \\
\hline & & -1 & -1 & 1 & 1 & 0 & 1 & 0 & 0 & 0 & -1 & 0 & c \\
\hline
\end{tabular}


Table 5

The combinations of 8 -link operators which yield the representations shown; $P$ takes the values \pm 1 .

\begin{tabular}{rrrrrrrrr}
\hline \hline $\mathcal{D}_{4}^{\mathrm{h}}$ & $T_{x y-x t}^{8 P}$ & $T_{y-x-y t}^{8 P}$ & $T_{-x-y x t}^{8 P}$ & $T_{-y x y t}^{8 P}$ & $T_{x-y-x t}^{8 P}$ & $T_{y x-y t}^{8 P}$ & $T_{-x y x t}^{8 P}$ & $T_{-y-x y t}^{8 P}$ \\
\hline$A_{3}^{P}$ & 1 & 1 & 1 & 1 & 1 & 1 & 1 & 1 \\
$B_{1}^{P}$ & 1 & -1 & 1 & -1 & 1 & -1 & 1 & -1 \\
$A_{2}^{P}$ & 1 & 1 & 1 & 1 & -1 & -1 & -1 & -1 \\
$B_{2}^{P}$ & 1 & -1 & 1 & -1 & -1 & 1 & -1 & 1 \\
$E^{P}$ & 1 & 0 & -1 & 0 & 0 & 1 & 0 & -1 \\
& 0 & 1 & 0 & -1 & -1 & 0 & 1 & 0 \\
& 1 & 0 & -1 & 0 & 0 & -1 & 0 & 1 \\
& 0 & 1 & 0 & -1 & 1 & 0 & -1 & 0 \\
\hline
\end{tabular}

$$
\begin{aligned}
P_{\mu \nu}^{4} & =\frac{1}{2 N} \operatorname{tr} U(\mu, \nu,-\mu,-\nu) & & \text { 4-link } \\
T_{\mu \nu \rho}^{6} & =\frac{1}{2 N} \operatorname{tr} U(\mu, \nu, \rho,-\mu,-\nu,-\rho) & & \text { twisted 6-link } \\
P_{\mu \mu \nu}^{6} & =\frac{1}{2 N} \operatorname{tr} U(\mu, \mu, \nu,-\mu,-\mu,-\nu) & & \text { planar 6-link } \\
B_{\mu \nu \rho}^{6} & =\frac{1}{2 N} \operatorname{tr} U(\mu, \nu, \rho,-\nu,-\mu,-\rho) & & \text { bent 6-link } \\
T_{\mu \nu-\mu \rho}^{8} & =\frac{1}{2 N} \operatorname{tr} U(\mu, \nu,-\mu,-\nu,-\mu, \rho, \mu,-\rho) & & \text { twisted plaquette pair. }
\end{aligned}
$$

The notation $U(\mu, \nu, \ldots)$ denotes a product of link matrices $U$ over a loop. The loop is specified from an arbitrary starting point by the directions $\mu, \nu$, etc., in order. The additional definitions

$$
\begin{aligned}
B_{\mu, t, \nu}^{6 \pm} & =B_{\mu, t, \nu}^{6} \pm B_{\mu,-t, \nu}^{6}, \\
T_{\mu, \nu,-\mu, t}^{8 \pm} & =T_{\mu, \nu,-\mu, t}^{8} \pm T_{\mu, \nu,-\mu,-t}^{8}
\end{aligned}
$$

turn out to be convenient. The $C=1$ representations are always obtained by taking the real part of the loop and the $C=-1$ representations by the imaginary part.

The tables can be used to get two related pieces of information. The entries are the coefficients for linear combinations of loop operators required to obtain the indicated representation of $\mathcal{D}_{4}^{\mathrm{h}}$. The combinations for 4-link operators are given in Table 1; for twisted 6-link operators in Table 2; for planar and bent 6-link operators in Tables 3 and 4 respectively; and finally for a set of 8-link operators in Table 5. For 4- and 6-link operators, the breakup of $\mathrm{O}_{\mathrm{h}}$ representations (the $T=$ 0 symmetry group) for these sets of operators into representations of $\mathcal{D}_{4}^{\mathrm{h}}$ are also specified. For the 8-link operators this decomposition is not given. In our later analysis we shall require this information for the $B_{1}^{++}$and $B_{2}^{++}$representations built out of these operators. In these two cases, the containing representations of $\mathrm{O}_{\mathrm{h}}$ are uniquely fixed to be $E^{++}$and $T_{2}^{++}$. 
Table 6

Irreducible components $D$ of twofold products $D_{1} \otimes D_{2}$ of representations of $\mathcal{D}_{4}^{\text {h }}$. Note that the $\mathrm{A}_{2}^{++}$state vector cannot be combined into a colourless symmetric state and is therefore forbidden.

\begin{tabular}{cll}
\hline$D_{1} \otimes D_{2}$ & \multicolumn{1}{c}{$D$} & \multicolumn{1}{c}{ State vector } \\
\hline$A_{1}^{--} \otimes A_{1}^{--}$ & $A_{1}^{++}$ & $A_{t} A_{t}$ \\
$A_{1}^{--} \otimes E^{+-}$ & $E^{-+}$ & $A_{l} A_{x}+A_{x} A_{t}$ \\
& & $A_{t} A_{y}+A_{y} A_{t}$ \\
$E^{+-} \otimes E^{+-}$ & $A_{1}^{++}$ & $A_{x}^{2}+A_{y}^{2}$ \\
& $\oplus B_{1}^{++}$ & $A_{x}^{2}-A_{y}^{2}$ \\
& $\oplus B_{2}^{++}$ & $A_{x} A_{y}+A_{y} A_{x}$ \\
& $\left(\oplus A_{2}^{++}\right)$ & $\left(A_{x} A_{y}-A_{y} A_{x}\right)$ \\
\hline
\end{tabular}

Table 7

Irreducible components $D$ of threefold products $D_{1} \otimes D_{2} \otimes D_{3}$ of representations of $\mathcal{D}_{4}^{\mathrm{h}}$.

\begin{tabular}{cl}
\hline$D_{1} \otimes D_{2} \otimes D_{3}$ & $D$ \\
\hline$A_{1}^{--} \otimes A_{1}^{--} \otimes A_{1}^{--}$ & $A_{1}^{--}$ \\
$A_{1}^{-} \otimes A_{1}^{-} \otimes E^{+-}$ & $E^{+-}$ \\
$A_{1}^{-} \otimes E^{+-} \otimes E^{+-}$ & $A_{1}^{--}+A_{2}^{--}+B_{1}^{--}+B_{2}^{--}$ \\
$E^{+-} \otimes E^{+-} \otimes E^{+-}$ & $4 E^{+-}$ \\
\hline
\end{tabular}

\subsection{Link operators and their direct products}

At high temperatures one may expect the observed correlations to be explained in terms of multiple gluon exchanges. Therefore it is necessary to perform a reduction of multigluon states to the irreps of $\mathcal{D}_{4}^{\mathrm{h}}$. In order to derive the corresponding selection rules we start from the definition of a gluon field on the lattice,

$$
A_{\mu}(x)=\frac{1}{2 i}\left[\left\{U_{\mu}(x)-U_{\mu}^{\dagger}(x)\right\}-\operatorname{tr}\left\{U_{\mu}(x)-U_{\mu}^{\dagger}(x)\right\}\right]
$$

for a gluon operator in any appropriately fixed gauge. Thus the electric gluon, $A_{t}$, transforms according to the irrep $A_{1}^{--}$of $\mathcal{D}_{4}^{\mathrm{h}}$ and the magnetic gluons, $A_{x}$ and $A_{y}$, according to the irrep $E^{+-}$of $\mathcal{D}_{4}^{\text {h }}$.

The reduction of the direct product representations is straightforward. For twogluon states the results are listed in Table 6. Since each gluon is a colour octet, there is only one symmetric combination of two gluons to a colour singlet. Hence the $A_{2}^{++}$state of Table 6 cannot be combined into a colourless state. However, the $A_{1}^{++}, E^{-+}, B_{1}^{++}$, and $B_{2}^{++}$are allowed as two gluon states. The reduction of the three-gluon states is listed in Table 7.

We note that the reduction of two- and three-gluon states in Tables 6 and 7 does not take into account the possible relative motion of the gluons. It thus applies directly only to gluons that are in an orbital s-state. We were able to observe an $A_{2}^{-+}$state (see section 4), which does not appear in Tables 6 and 7 . It is easy to see that it can be built of two gluons in an orbital p-state. 
Table 8

Lattice sizes $N_{\tau} \times N_{\sigma}^{3}$, Wilson coupling $\beta$, number of measurements $N_{\mathrm{m}}$ and size $N_{\mathrm{b}}$ of one jackknife bin for analysis.

\begin{tabular}{ccrr}
\hline$N_{\tau} \times N_{\sigma}^{3}$ & $\beta$ & $N_{\mathrm{m}}$ & $N_{\mathrm{b}}$ \\
\hline $4 \times 16^{3}$ & 5.93 & 13000 & 1300 \\
$8 \times 16^{3}$ & 5.93 & 5000 & 500 \\
$6 \times 16^{3}$ & 6.101 & 6300 & 630 \\
\hline
\end{tabular}

The two possible channels in the $A_{1}^{++}$sector can thus be separated by splitting operators into two groups-those containing links in the time direction, and those that do not. These two sets should give, in general, unequal screening masses. In the $g(T) \rightarrow 0$ limit, these screening masses are then equal to $2 M_{\mathrm{e}}$ and $2 M_{\mathrm{m}}$. In this limit the $B_{1}^{++}$and $B_{2}^{++}$screening masses should also yield $2 M_{\mathrm{m}}$; although for any finite $T$ there should be splittings between these three screening masses. Similarly, the $A_{2}^{-+}$masses should be asymptotically degenerate with these masses. In the $T \rightarrow \infty$ limit, obvious cross checks should be provided by the screening masses in the $E^{-+}$and the negative $C$-parity channels.

\section{Simulations and measurements}

Simulations were carried out in pure SU (3) gauge theory at two temperatures. One was chosen to be in the confined phase, at about $0.75 T_{\mathrm{c}}$, and the other in the deconfined phase, at about $1.5 T_{\mathrm{c}}$. Since the phase transition for $N_{\tau}=6$ occurs at $\beta_{c} \simeq 5.9$ [14], we chose to work with $8 \times 16^{3}$ and $4 \times 16^{3}$ lattices at fixed $\beta=5.93$. Scaling was investigated by a separate run on a $6 \times 16^{3}$ lattice at $\beta=6.101$. Both the $N_{\tau}=4$ and 6 lattices were at the same physical temperature in the deconfined phase. Further details of the runs are listed in Table 8.

The simulations were performed with an $S U(2)$ subgroup over-relaxation (OR) mixed with a Cabbibo-Marinari pseudo-heatbath (HB) update [15]. We used 4 steps of OR to each of HB. The HB step used a Kennedy-Pendleton SU(2) heatbath [16]. The time for $4 \mathrm{OR}+1 \mathrm{HB}$ updates is $115 \mu$ s per link on a Cray Y-MP. Measurements were performed on configurations separated by 50 HB steps. The auto-correlations times away from $T_{\mathrm{c}}$ are expected to be rather small, and each of these configurations is essentially uncorrelated with the others.

\subsection{Fuzzed operators}

Past experience, at $T=0$, indicates that the signal to noise ratio for looploop correlations decays extremely fast with separation. It seems necessary to use a signal enhancement technique in order to measure correlation functions at distances greater than 2 or 3 lattice spacings. Accordingly, we used loops constructed out of fuzzed links [17]. This is a well-known technique. For each configuration, the link matrices generated are considered to be the level $l=0$ 
of fuzzing. Then a link $U_{\mu}^{l}(i)$ at fuzzing level $l$ is given in terms of those at the previous level, $l-1$, by the relation

$$
\begin{aligned}
& U_{\mu}^{l}(i)=\left[U_{\mu}^{l-1}(i) U_{\mu}^{l-1}(i+\mu)\right. \\
& \left.+\sum_{\nu \neq \mu} U_{\nu}^{l-1}(i) U_{\mu}^{l-1}(i+\nu) U_{\mu}^{l-1}(i+\nu+\mu)\left(U_{\nu}^{l-1}(i+2 \mu)\right)^{\dagger}\right]_{\mathrm{SU}(3)},
\end{aligned}
$$

where the subscript denotes a projection onto $\mathrm{SU}(3)$. This projection is obtained through the polar decomposition of the general complex $3 \times 3$ matrix, $M$, on the right: $M=\omega U H$. $H$ is a hermitian matrix, $\omega$ a complex number and $U$ the required special unitary matrix ${ }^{\star}$. We let the indices $\mu$ and $\nu$ in Eq. (7) range over the $x, y$ and $t$ directions. In each direction we perform fuzzing up to level $\left(\log _{2} N-1\right)$, where $N$ is the lattice size. Thus, for $N_{\tau}=4$ we perform fuzzing up to level 1 for links in the $t$ direction.

We constructed the operators listed in section 2 out of the fuzzed links obtained by this procedure. Note also that the exclusion of the $z$-direction links from the sum in Eq. (7) implies that the separation in the correlation functions measured is a well-defined quantity. Further, since the $z$ links do not enter any of the operators we study, the loss in fuzzing is probably not very important.

\subsection{Mass measurements}

Since different operators realising the same representation of $\mathcal{D}_{4}^{\text {h }}$ can be correlated through the exchange of the same state, we have measured all elements of the full correlation matrix

$$
C_{\alpha, \beta}(z)=\left\langle L_{\alpha}(0) L_{\beta}(z)\right\rangle-\left\langle L_{\alpha}\right\rangle\left\langle L_{\beta}\right\rangle,
$$

where $\alpha$ and $\beta$ label the loop operators in a given symmetry sector. The loop operators were summed over all sites on a $z$-slice, in order to project onto zero momentum. We can obtain the lowest lying excitation by a variational procedure, see for instance ref. [20]. This involves solving the generalised eigenvalue problem

$$
C_{\alpha, \beta}\left(z_{1}\right) Y_{\beta}=\lambda\left(z_{1}, z_{0}\right) C_{\alpha, \beta}\left(z_{0}\right) Y_{\beta} .
$$

Then the quantity

$$
g(z)=Y_{\alpha} C_{\alpha, \beta}(z) Y_{\beta},
$$

* Though $U$ might not always be the "nearest" $S U(3)$ matrix to $M$ [18], the polar decomposition is fast and for our purposes sufficient. Another, more elaborate procedure, would be to maximise $\operatorname{Re} \operatorname{Tr}\left(U^{\dagger} M\right)$ iteratively [19]. 
where $Y$ belongs to the lowest eigenvalue, projects onto the correlation function with the smallest mass. Local masses, $m(z)$, were extracted by solving the equation

$$
\frac{g(z)}{g(z-1)}=\frac{\cosh \left[m(z)\left(N_{z} / 2-z\right)\right]}{\cosh \left[m(z)\left(N_{z} / 2-(z-1)\right)\right]},
$$

where the left-hand side was obtained from the measurements. Jackknife estimators were used for the local masses and their errors. If the diagonalisation procedure exactly isolates the lowest mass into $g(z)$, then $m(z)$ should be independent of $z$ within errors. In practice this does not happen, and we have to identify the lowest mass through a plateau in $m(z)$. The effectiveness of the diagonalisation procedure is nevertheless seen in the extension of this plateau region.

We restricted ourselves to diagonalising for $z_{1}=1$ and 2 at $z_{0}=0$ in order to determine the leading eigenvector $Y$ for each set of quantum numbers. The two different sets of eigenvectors were found to be reasonably similar. It is useful to recall that this eigenvector gives the weight of each loop to the lowest state, and hence is an analogue of the modulus squared of the "wavefunction".

\subsection{Polyakov loops}

We have also measured the zero-momentum correlation functions between Polyakov loops

$$
C_{\mathrm{ww}}(z)=\left\langle\Omega^{\dagger}(z) \Omega(0)\right\rangle-\left\langle\Omega^{\dagger}\right\rangle\langle\Omega\rangle .
$$

The zero-momentum projection is achieved through the use of the walls

$$
\Omega(z)=\sum_{x y} \Omega(x, y, z), \quad \Omega(x, y, z)=\operatorname{tr} \prod_{t} U_{0}(x, y, z, t) .
$$

The conjugate wall operator $\Omega^{\dagger}$ is defined by replacing the link operators in Eq. (13) by their hermitian conjugates. The static potential $V(r)$ between a quark and an antiquark at distance $\boldsymbol{r}=(x, y, z)$ is usually measured through point to point correlation functions in the form

$$
\exp (-V(\boldsymbol{r}) / T)=\frac{\left\langle\Omega^{\dagger}(\boldsymbol{r}) \Omega(0)\right\rangle}{\left\langle\Omega^{\dagger}\right\rangle\langle\Omega\rangle} .
$$

In perturbation theory the colour averaged potential above is expected to have the form $\exp \left(-M_{\mathcal{P}} r\right) / r^{2}$. Expanding the exponential in Eq. (14) and substituting the perturbative form for the potential, one can then derive the appropriate form for $C_{\mathrm{Ww}}$. In the long distance limit it is given by the exponential integral $E_{1}\left(M_{\mathcal{P}} z\right)$. We actually use the symmetrised form

$$
C_{\mathrm{ww}}(z) \propto E_{1}\left(M_{\mathcal{P}} z\right)+E_{1}\left(M_{\mathcal{P}}\left(N_{z}-z\right)\right),
$$

and determine $M_{\mathcal{P}}$ by an appropriate generalisation of a local mass. 
In the confining phase, $V(r) \propto r K_{\text {eff }} / T$. The appropriate symmetrised form for the zero-momentum correlation function is then

$$
C_{\mathrm{ww}}(z)=A \cosh \left[\frac{K_{\mathrm{eff}}}{T}\left(\frac{N_{z}}{2}-z\right)\right],
$$

where $A$ is some constant. It is clear that a local mass measurement yields $K_{\text {eff }} / T$ and hence a plateau in this can be used for a measurement of the string tension at finite temperatures.

The correlation function

$$
C_{\mathrm{aa}}(z)=\left\langle\left|\Omega^{\dagger}(z) \| \Omega(0)\right|\right\rangle-\left\langle\left|\Omega^{\dagger}\right|\right\rangle\langle|\Omega|\rangle,
$$

on the other hand, has trivial triality, and hence furnishes an alternative measurement of the mass in the $A_{1}^{++}$channel.

\section{Results}

The local masses obtained from eqs. (9) through (11) are listed in Table 9. We have underlined the values which we consider to be the best estimates of the asymptotic masses. In all our measurements an $A_{1}^{++}$mass turned out to be the lowest. The corresponding correlation functions were the least noisy, and the local masses could be followed out to distance 5 or 6 . In this case it seems quite likely that we are able to identify the lowest mass in this channel. Apart from this, local masses at distance greater than 1 could only be measured with reasonable accuracy in the channels with quantum numbers $A_{2}^{-+}, B_{1}^{++}$and $B_{2}^{++}$. In these cases we could follow the local masses up to about distance 3 . It is likely that the extraction of a mass in these latter channels is contaminated by the presence of higher states. There is more structure in our measurements than is seen in the group theory presented in section 2 . This is good news, since it indicates nontrivial dynamics.

\subsection{The $A_{1}^{++}$channel}

Note that the $A_{1}^{++}$mass obtained through the trivial-triality correlations of Polyakov loops is always equal to the lowest mass in the $A_{1}^{++}$channel extracted using loop operators. This provides a cross check on the proper extraction of this mass.

Below $T_{\mathrm{c}}$ this channel descends from the $E^{++}$and $A_{1}^{++}$representations of the $\mathrm{O}_{\mathrm{h}}$ group. Grouping the operators constituting this representation of $\mathcal{D}_{4}^{\mathrm{h}}$ into two sets, one for each of the $T=0$ symmetries, we find that the lowest mass in the former group is about twice that in the latter. Such a splitting is also indicated by the eigenvectors, $Y_{\beta}$, defined in Eq. (9). The low-temperature system thus seems to realise the $T=0$ symmetries dynamically. 
Table 9

Local masses, $m(z)$, for the different representations tabulated as a function of $z$. We take the underlined values as our best estimate for the asymptotic masses. In the confined phase $\left(N_{\tau}=8\right)$ we list the $\mathrm{O}_{\mathrm{h}}$ representation to which the operator belongs, while in the deconfined phase $\left(N_{\tau}=4\right.$ and 6) we indicate for the representation whether the operator contains time-like links or not.

\begin{tabular}{|c|c|c|c|c|c|c|c|c|c|c|}
\hline \multirow[t]{2}{*}{$N_{\tau}$} & \multirow[t]{2}{*}{$\beta$} & \multirow[t]{2}{*}{ Rep } & \multirow[t]{2}{*}{ Operator } & \multirow[t]{2}{*}{$l$} & \multicolumn{6}{|c|}{$z$} \\
\hline & & & & & 1 & 2 & 3 & 4 & 5 & 6 \\
\hline \multirow[t]{6}{*}{8} & \multirow[t]{6}{*}{5.93} & $A_{1}^{++}$ & local, $A_{1}^{++}$ & 2 & $0.87(1)$ & $0.78(2)$ & $0.77(4)$ & $0.7(1)$ & $0.46(8)$ & $0.5(2)$ \\
\hline & & $A_{1}^{++}$ & local, $E^{++}$ & 2 & $1.39(2)$ & $1.07(5)$ & $1.0(2)$ & $0.6(3)$ & & \\
\hline & & $A_{1}^{++}$ & $C_{\mathrm{aa}}$ & 2 & $1.23(2)$ & $0.63(3)$ & $\overline{0.58(5)}$ & $0.51(8)$ & $0.6(1)$ & $0.6(2)$ \\
\hline & & $\mathrm{A}_{2}^{-+}$ & local & 2 & $1.84(2)$ & $1.6(1)$ & $1.2(5)$ & & & \\
\hline & & $B_{1}^{++}$ & local, $E^{++}$ & 2 & $1.38(2)$ & $1.21(6)$ & $\overline{1.0(1)}$ & $0.8(3)$ & & \\
\hline & & $B_{2}^{++}$ & local, $T_{2}^{++}$ & 2 & $1.45(2)$ & $1.27(6)$ & $1.2(1)$ & $1.2(4)$ & & \\
\hline \multirow[t]{5}{*}{4} & \multirow[t]{5}{*}{5.93} & $A_{1}^{++}$ & local, t & 2 & $1.012(6)$ & $0.80(3)$ & $0.73(5)$ & $0.8(1)$ & $0.7(3)$ & \\
\hline & & $A_{1}^{++}$ & local, no $t$ & 2 & $1.36(1)$ & $1.27(4)$ & $\overline{1.3(1)}$ & $0.7(2)$ & & \\
\hline & & $A_{2}^{-+}$ & local & 2 & $1.54(1)$ & $1.12(3)$ & $\overline{1.0(1)}$ & $0.8(2)$ & & \\
\hline & & $B_{1}^{++}$ & local & 2 & $2.22(2)$ & $2.0(2)$ & & & & \\
\hline & & $B_{2}^{++}$ & local & 2 & $2.37(2)$ & $1.9(2)$ & & & & \\
\hline \multirow[t]{6}{*}{6} & \multirow[t]{6}{*}{6.101} & $\mathrm{~A}_{1}^{++}$ & local, t & 2 & $0.97(1)$ & $0.64(4)$ & $0.61(8)$ & $0.51(13)$ & $0.43(11)$ & \\
\hline & & $A_{1}^{++}$ & local, no $\mathfrak{t}$ & 2 & $1.063(7)$ & $0.96(2)$ & $0.97(7)$ & $1.1(2)$ & & \\
\hline & & $A_{1}^{++}$ & $C_{\mathrm{aa}}$ & 0 & $1.0(1)$ & $0.56(1)$ & $0.46(2)$ & $0.51(4)$ & $0.49(7)$ & $0.48(6)$ \\
\hline & & $A_{2}^{-+}$ & local & 2 & $1.40(2)$ & $1.00(3)$ & $0.83(6)$ & $0.8(2)$ & & \\
\hline & & $\mathrm{B}_{1}^{++}$ & local & 2 & $1.82(1)$ & $1.6(1)$ & $1.3(2)$ & & & \\
\hline & & $B_{2}^{++}$ & local & 2 & $1.82(2)$ & $1.57(7)$ & $\overline{1.5(5)}$ & & & \\
\hline
\end{tabular}

This is most easily demonstrated by the eigenvectors restricted to the twodimensional space spanned by plaquette correlations, where the plaquette operators could have a link in the euclidean-time direction $\left(P_{t}=P_{x t}^{4}+P_{y l}^{4}\right)$ or no links in this direction $\left(P_{s}=P_{x y}^{4}\right)$. An operator in the $E^{++}$representation of $\mathrm{O}_{\mathrm{h}}$ can be formed by the combination $-2 P_{s}+P_{t}$. We denote this in the shorthand notation $(-2,1)$. The $A_{1}^{++}$representation of $\mathrm{O}_{\mathrm{h}}$ is given by $(1,1)$. Diagonalisation of the correlation matrix in this subspace gives the lowest mass of $0.5 \pm 0.1$ with the corresponding eigenvector equal to $(0.91 \pm 0.03,1.00 \pm 0.03)$. The next mass is roughly 1.0 and the eigenvector corresponding to this is $(-2.093 \pm 0.007,1.00 \pm$ 0.02 ). Similar results are obtained in the three-dimensional subspace of planar 6-link operators.

Since the $T=0$ symmetries seem to be generated dynamically, it is of interest to ask about the temperature dependence of glueball masses below $T_{\mathrm{c}}$. Our best estimate for the $A_{1}^{++}\left(\mathrm{O}_{\mathrm{h}}\right.$ representation $)$ mass at $T=0.75 T_{\mathrm{c}}$ is $0.49(8)$. This mass has been measured at $T=0$ [21] to be 0.81 (3) at $\beta=5.93$ on a $24^{3} \times 36$ lattice. Measurements at $\beta=5.90$ on $12^{4}$ lattices verify that finite size effects near this coupling are small. Our measurement yields 


$$
\frac{m\left(T=0.75 T_{\mathrm{c}}\right)}{m(T=0)}=0.6 \pm 0.1 \quad\left(A_{1}^{++}, \beta=5.93\right) .
$$

Thus, the thermal shift is much larger than that seen in quenched hadron masses [22].

Above $T_{\mathfrak{c}}$, the situation is quite different. There is no evidence of a splitting of the $A_{1}^{++}$level according to the $T=0$ symmetry. On the other hand, a definite splitting is observed when the operators are grouped into two sets, one with loops containing no links in the $t$ direction, and the other containing at least one such link. From the decomposition of the multi-gluon states (see Table 6) it is seen that these two sets correspond to the exchange of two magnetic and two electric gluons respectively. This interpretation is further supported by the agreement of the second of these two masses with $M_{\mathcal{P}}$. It should be noted, however, that loops containing both space-like and time-like links can couple to magnetic and electric gluons with the lighter ones dominating the correlation function at large distances. For our parameters the electric gluons seem to be lighter and hence dominating.

The eigenvectors also give evidence of such a decomposition. The two dimensional space of plaquette correlations for the $N_{\tau}=6$ lattice again demonstrates this very well. The lowest mass corresponds to the eigenvector $(0.2 \pm 0.2,1.01 \pm$ 0.05 ), i.e. to an operator with large overlap with $P_{t}$. The next eigenvector seems to have a larger overlap with $P_{s}$ than with $P_{t}$. Similar results can also be obtained in the three-dimensional space of planar 6-link operators. Finally, note that $m / T$ is the same for the $N_{\tau}=4$ and 6 lattices, showing that finite lattice-spacing effects are under control.

\subsection{Polyakov loops}

The results for the effective string tension $K_{\text {eff }}$ below $T_{\mathrm{c}}$ and the mass $M_{\mathcal{P}}$ above $T_{\mathrm{c}}$ are listed in Table 10. For the effective string tension at a temperature $T=0.75 T_{\mathrm{c}}$ we find $\sqrt{K_{\text {eff }}(T)}=0.197(6)$. Although there is no measurement of $\sqrt{K_{\text {eff }}}$ at precisely this coupling at $T=0$, it is possible to estimate this value from an interpolation of the data given in ref. [21]. We find

$$
\sqrt{\frac{K_{\text {eff }}\left(T=0.75 T_{\mathrm{c}}\right)}{K_{\text {eff }}(T=0)}}=0.83(4)
$$

As a consequence, we find that the ratio $m\left(A_{1}^{++}\right) / \sqrt{K_{\text {eff }}}$ is itself temperature dependent, changing from 3.5(2) at $T=0$ to a value of $2.5(5)$ at $T=0.75 T_{\mathrm{c}}$.

The simulations for $N_{\tau}=4$ and 6, corresponding to a temperature of about $1.5 T_{\mathrm{c}}$, yield consistent values for $M_{\mathcal{P}} / T$. From the data in the table, it is seen that we obtain

$$
M_{\mathcal{P}}=2.0(2) T \text { at } N_{\tau}=4 \quad \text { and } \quad M_{\mathcal{P}}=2.0(4) T \text { at } N_{\tau}=6 .
$$


Table 10

Local values of $K_{\text {eff }}$ for $N_{\tau}=8$ and $M_{\mathcal{P}}$ for $N_{\tau}=4$ and 6 .

\begin{tabular}{|c|c|c|c|c|c|c|c|c|c|}
\hline \multirow[t]{2}{*}{$N_{t}$} & \multirow[t]{2}{*}{$\beta$} & \multirow[t]{2}{*}{$l$} & \multicolumn{7}{|c|}{$z$} \\
\hline & & & 1 & 2 & 3 & 4 & 5 & 6 & 7 \\
\hline 8 & 5.93 & 2 & $0.071(1)$ & $0.043(1)$ & $0.041(1)$ & $0.040(3)$ & $0.039(3)$ & $0.036(4)$ & $0.043(3)$ \\
\hline 4 & 5.93 & 0 & & $0.400(8)$ & $0.47(3)$ & $0.51(5)$ & $\overline{0.66(12)}$ & $0.5(2)$ & \\
\hline 6 & 6.101 & 0 & & $0.241(8)$ & $0.24(1)$ & $\overline{0.34(4)}$ & $0.34(6)$ & $0.33(6)$ & $0.3(1)$ \\
\hline
\end{tabular}

These should be compared to the value $1.63(8)$, obtained at $2 T_{\mathrm{c}}$ in [23] from measurements of the static inter-quark potential, and the one-loop perturbation theory result $2 M_{\mathrm{e}} / T=2 g(T)$ where $g(T) \approx 1$.

\subsection{Other channels}

At $T=0$ the measurement of excited glueball masses is not easy. Nor does a finite temperature render this measurement any easier. Correlation functions in channels other than $A_{1}^{++}$cannot be followed very far. As a result, evidence for a genuine plateau in the local masses is not conclusive. Although we are able to tentatively assign screening masses to correlations in these channels, more detailed work will be necessary to achieve the reliability obtained in the $A_{1}^{++}$ channel.

A very interesting phenomenon is the observation of non-trivial correlations in the $A_{2}^{-+}$sector. Asymptotically in $T$ this mass should be given by

$$
m_{A_{2}^{-+}} \rightarrow 2 \sqrt{M_{\mathrm{e}}^{2}+\sin ^{2}\left(\frac{2 \pi}{N}\right)},
$$

where $2 \pi / N$ is the lowest non-zero mode for an electric gluon. This goes to $M_{\mathcal{P}}$ only in the limit of the spatial volume going to infinity $(N \rightarrow \infty)$. This formula works surprisingly well even at the temperature we study. For our lattice sizes $(N=16)$, taking $M_{\mathrm{e}}$ to be half the lowest $A_{1}^{++}$mass, we find $m_{A_{2}^{-+}} \approx 0.90$ for $N_{\tau}=6$ and 1.06 for $N_{\tau}=4$.

It is also interesting that below $T_{\mathrm{c}}$ the $A_{1}^{++}$mass originating from the $E^{++}$representation of $\mathrm{O}_{\mathrm{h}}$ seems to be degenerate with the $B_{1}^{++}$mass originating from the same representation. This is consistent with other evidence that the zerotemperature spectrum is realised dynamically in this phase. Further investigation of these other glueball channels, at a sequence of temperatures, would be rewarding.

\section{Conclusion}

In this paper we have presented first measurements of screening masses for pure-glue operators at high temperatures in SU (3) gauge theories. We have iden- 
tified the symmetry of the " $z$-slice group", $\mathcal{D}_{4}^{\mathrm{h}}$, and presented the reduction of loop operators under this symmetry. In order to understand the dynamics giving rise to the measured screening masses, we have also performed the reduction of these representations under the symmetries of the zero-temperature theory. Furthermore, we have examined the symmetries of the link operators, "gluons", and performed the reduction of direct products of these representations.

Correlations in the $A_{1}^{++}$channel were the least noisy and, hence, the easiest to study. We were able to reach a detailed understanding of the dynamics in this channel. Our observations are completely consistent with the usual picture of a deconfinement phase transition in QCD. In the low-temperature phase one finds a (strong) perturbation of the zero temperature spectrum, whereas the high-temperature phase can be understood in terms of multi-gluon exchanges. Of course, as is well-known, at temperatures of $1.5 T_{\mathrm{c}}$, where our simulations were performed, the deconfined theory is not weakly interacting.

In the confined phase the correlation functions were seen to dynamically reproduce the symmetries of the zero-temperature theory. We observed this both in the spectrum and the "wavefunctions"--the lowest mass in the $A_{1}^{++}$channel (of $\mathcal{D}_{4}^{\mathrm{h}}$ ) originated from the correlations of operators transforming under the $\mathrm{A}_{1}^{++}$ channel of the symmetry group of the zero-temperature theory, and the next excited state in this channel could be identified as the correlations of the operators transforming as the $E^{++}$representation. As a result it is possible to talk of thermal shifts in glueball masses for $T<T_{\mathrm{c}}$. We found that at $T=0.75 T_{\mathrm{c}}$ the mass of the $0^{++}$glueball dropped to about half its zero-temperature value. This is a larger mass shift than anything seen in the meson spectrum.

The dynamical behaviour of these correlations in the deconfined phase are completely different. Both the mass-spectrum and the "wavefunctions" indicate a splitting between correlations carried by the electric and magnetic polarisations. Group theoretically, the $A_{1}^{++}$representation of $\mathcal{D}_{4}^{\text {h }}$ can arise from either two electric or two magnetic gluon exchanges in a relative s-wave state. Thus, to $\mathcal{O}\left(g^{2}(T)\right)$, the screening mass in this channel gives either $2 M_{\mathrm{e}}$ or $2 M_{\mathrm{m}}$. Such a splitting is seen in the spectrum, and is correlated with the use of operators with and without time-like links. Furthermore, the $A_{1}^{++}$screening mass in the electric sector agrees very well with the measured Wilson-line screening mass. Hence, we can write

$$
\begin{aligned}
& m_{A_{1}^{++}}=2.8(4) T \approx 2 M_{\mathrm{e}}, \\
& m_{A_{1}^{++}}^{\prime}=5.8(4) T \approx 2 M_{\mathrm{m}} \quad\left(T=1.5 T_{\mathrm{c}}\right) .
\end{aligned}
$$

These statements come with the usual caveat. The non-perturbative measurements of the two different kinds of screening masses in the $A_{1}^{++}$channel are valid at all temperatures, but the identification of these with twice the gluon electric and magnetic masses is valid only at high temperatures.

Within the context of perturbation theory, corrections to the identifications made above arise from multi-gluon exchanges. Since direct products of three glu- 
ons do not contain $A_{1}^{++}$, the $\mathcal{O}\left(g^{3}(T)\right)$ corrections can be obtained by resummation. Of course, these and higher corrections are not negligible at the couplings where our simulations are performed. Such corrections result in the mixing of electric and magnetic channels, as is seen easily when the perturbative diagrams are written out. Similar arguments may explain the fact, a little surprising at first sight, that $M_{\mathrm{m}}$ turns out to be larger than $M_{\mathrm{e}}$. Their ratio is expected to be proportional to $g(T)$, but we work at rather large values of $g(T)$; it is entirely possible that at higher temperatures, and hence weaker couplings, one obtains a different ordering of these masses.

The $A_{2}^{-+}$channel is correlated through similar two-gluon exchanges in a relative p-wave state. Thus, in perturbation theory at order greater than $g^{2}(T)$ the screening mass is split from the $A_{1}^{++}$channel. On lattices of finite spatial volumes this screening mass is split from the $A_{1}^{++}$even in the limit $g(T) \rightarrow 0$. Surprisingly, even for our simulations at $1.5 T_{\mathrm{c}}$, the observed splitting between the screening masses in these two channels seems to be due entirely to this finite volume effect. It would be extremely interesting to continue these measurements to higher temperatures.

A cross check on a perturbative interpretation would be provided by an unambiguous measurement of the $B_{1}^{++}$and $B_{2}^{++}$screening masses. These are also correlated by the exchange of two magnetic gluons, and hence should be (asymptotically) degenerate with the larger mass measured in the $A_{1}^{++}$channel. Present measurements do not support this strongly, but measurements at larger physical distances and at higher temperatures are clearly needed. We found the negative $C$-parity correlations extremely noisy. Better techniques for signal enhancement in these channels are clearly desirable, since the leading contributions to these are due to three-gluon exchanges.

In summary, we have performed measurements of screening masses in the pure gauge sector of QCD at finite temperatures, and identified the main change in dynamics across the phase transition temperature. In the low temperature phase we have measured the thermal shift in the $0^{++}$glueball mass. In the deconfined phase we have been able to identify screening masses which, in the high-temperature limit, measure electric and magnetic gluon masses, and seen that they are not degenerate. In a sense, these define "non-perturbative" gluon masses. Further studies of these screening masses are clearly of major interest.

\section{Acknowledgement}

The work of U.M.H. was supported in part by the DOE under grants DE-FG0585ER250000 and DE-FG05-92ER40742. The research of F.K. was supported in part by the National Science Foundation under Grant No. PHY89-04035 and the German Research Foundation (DFG) under Grant Pe 340/3-2. U.M.H. and F.K. would like to thank the Institute for Theoretical Physics at UCSB for the kind hospitality extended to them while the paper was in the finishing stages. We would like to thank Uwe-Jens Wiese for providing us with the updating 
routines used to generate the gauge field configurations. The computations have been performed at the Supercomputer Center HLRZ in Jülich and at the RWTH Aachen.

\section{References}

[1] C. DeTar and J. Kogut, Phys. Rev. Lett. 59 (1987) 399;

S. Gottlieb et al., Phys. Rev. Lett. 59 (1987) 1881;

A. Gocksch, P. Rossi and U. M. Heller, Phys. Lett. B205 (1988) 334

[2] K. D. Born et al., Phys. Rev. Lett. 67 (1991) 302

[3] S. Gupta, Phys. Lett. B283 (1992) 439;

G. Boyd et al., HLRZ preprint HLRZ/54-93 (1993)

[4] G. Boyd, S. Gupta and F. Karsch, Nucl. Phys. B385 (1992) 481

[5] C. Bernard et al., Phys. Rev. Lett. 68 (1992) 2125

[6] V. Koch et al., Phys. Rev. D46 (1992) 3169

[7] C. Borgs, Nucl. Phys. B261 (1985) 455;

E. Manousakis and J. Polonyi, Phys. Rev. Lett. 58 (1987) 847;

L. Kärkkainen et al., Phys. Lett. B312 (1993) 173;

G. Bali et al., Phys. Rev. Lett. 71 (1993) 3059

[8] D. J. Gross, R. D. Pisarski, and L. G. Yaffe, Rev. Mod. Phys. 53 (1981) 43

[9] R. Kobes, G. Kunstatter and A. Rebhan, Phys. Rev. Lett. 64 (1990) 2992

[10] S. Nadkarni, Phys. Rev. D33 (1986) 3738; D34 (1986) 3904

[11] P. Bacilieri et al., Phys. Lett. B220 (1989) 607

[12] G. Lazarides, A. Billoire and Q. Shafi, Phys. Lett. B103 (1981) 450;

T. A. DeGrand and C. DeTar, Phys. Rev. D25 (1982) 526;

G. Lazarides and S. Sarantakos, Phys. Rev. D31 (1985) 389

[13] B. Berg and A. Billoire, Nucl. Phys. B221 (1983) 109

[14] Y. Iwasaki et al., Phys. Rev. D46 (1992) 4657

[15] N. Cabibbo and E. Marinari, Phys. Lett. B119 (1982) 387

[16] A. D. Kennedy and B. J. Pendleton, Phys. Lett. B156 (1985) 393

[17] M. Teper, Phys. Lett. B183 (1986) 345

[18] R. Gupta et al., Phys. Rev. Lett. 53 (9184) 1721

[19] C. Michael and M. Teper, Phys. Lett. B206 (1988) 299

[20] M. Lüscher and U. Wolff, Nucl. Phys. B339 (1990) 222

[21] C. Michael and M. Teper, Nucl. Phys. B314 (1989) 347;

Y. Liang et al., Univ. of Kentucky preprint UK/92-05 (1992);

H. Chen et al., preprint hep-lat 9308010 (1993)

[22] G. Boyd et al., work in progress; a preliminary report may be found in S. Gupta, HLRZ preprint HLRZ 53-94 (hep-ph 9308319)

[23] L. Kärkkäinen et al., Phys. Lett. B282 (1992) 121 